\title{
A multilevel study of area socio-economic status and food purchasing behaviour
}

\author{
Gavin Turrell ${ }^{1, *}$, Rebecca Bentley², Lyndal R Thomas², Damien Jolley33, \\ SV Subramanian ${ }^{4}$ and Anne M Kavanagh ${ }^{2}$ \\ ${ }^{1}$ School of Public Health, Institute for Health and Biomedical Innovation, Queensland University of Technology, \\ Victoria Park Road, Kelvin Grove, Brisbane, Queensland 4059, Australia: ${ }^{2}$ Key Centre for Women's Health in \\ Society, University of Melbourne, Melbourne, Victoria, Australia: ${ }^{3}$ Monash Institute of Health Services Research, \\ Monash University, Melbourne, Victoria, Australia: ${ }^{4}$ Department of Society, Human Development and Health, \\ Harvard School of Public Health, Boston, MA, USA
}

Submitted 19 July 2007: Accepted 11 December 2008: First published online 26 February 2009

\begin{abstract}
Objective: The present study examined the association between area socioeconomic status (SES) and food purchasing behaviour.

Design: Data were collected by mail survey (64.2\% response rate). Area SES was indicated by the proportion of households in each area earning less than \$AUS 400 per week, and individual-level socio-economic position was measured using education, occupation and household income. Food purchasing was measured on the basis of compliance with dietary guideline recommendations (for grocery foods) and variety of fruit and vegetable purchase. Multilevel regression analysis examined the association between area SES and food purchase after adjustment for individual-level demographic (age, sex, household composition) and socioeconomic factors.

Setting: Melbourne city, Australia, 2003.

Subjects: Residents of 2564 households located in fifty small areas.

Results: Residents of low-SES areas were significantly less likely than their counterparts in advantaged areas to purchase grocery foods that were high in fibre and low in fat, salt and sugar; and they purchased a smaller variety of fruits. There was no evidence of an association between area SES and vegetable variety. Conclusions: In Melbourne, area SES was associated with some food purchasing behaviours independent of individual-level factors, suggesting that areas in this city may be differentiated on the basis of food availability, accessibility and affordability, making the purchase of some types of foods more difficult in disadvantaged areas.
\end{abstract}

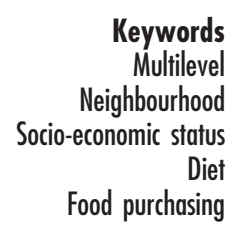

A large literature has examined the association between individual-level socio-economic position (SEP) and diet. The findings of this work typically show that socio-economically disadvantaged groups have diets that are least consistent with recommended intakes of foods and nutrients ${ }^{(1-3)}$ and least in accord with dietary guideline messages promoting foods that are high in fibre and low in fat, salt and sugar ${ }^{(4,5)}$. Significantly, the poorer dietary intake of disadvantaged groups contributes in part to their higher rates of mortality and morbidity for chronic disease $^{(6,7)}$.

During the last decade, researchers have increasingly turned their attention to the question of whether place of residence influences diet independently of individuallevel factors; and more particularly, whether living in a socio-economically disadvantaged area is associated with a less healthy diet. Our review of this (small) literature suggested that area studies of diet can be broadly divided into two types that reflect the analytical method used: (i) studies that undertake multivariable analyses using both area- and individual-level variables but without the capacity to statistically integrate the two levels (i.e. contextual studies); and (ii) multilevel studies. Six of the former types of study ${ }^{(8-13)}$ and five of the latter were identified $^{(14-18)}$, and key aspects of each are summarised in Table 1.

The findings of the contextual studies were reasonably consistent in that they each found some evidence that living in a disadvantaged area was associated with a poorer diet after adjusting for individual-level socio-economic and demographic factors. However, these studies were often based on a small number of areas and, in most 


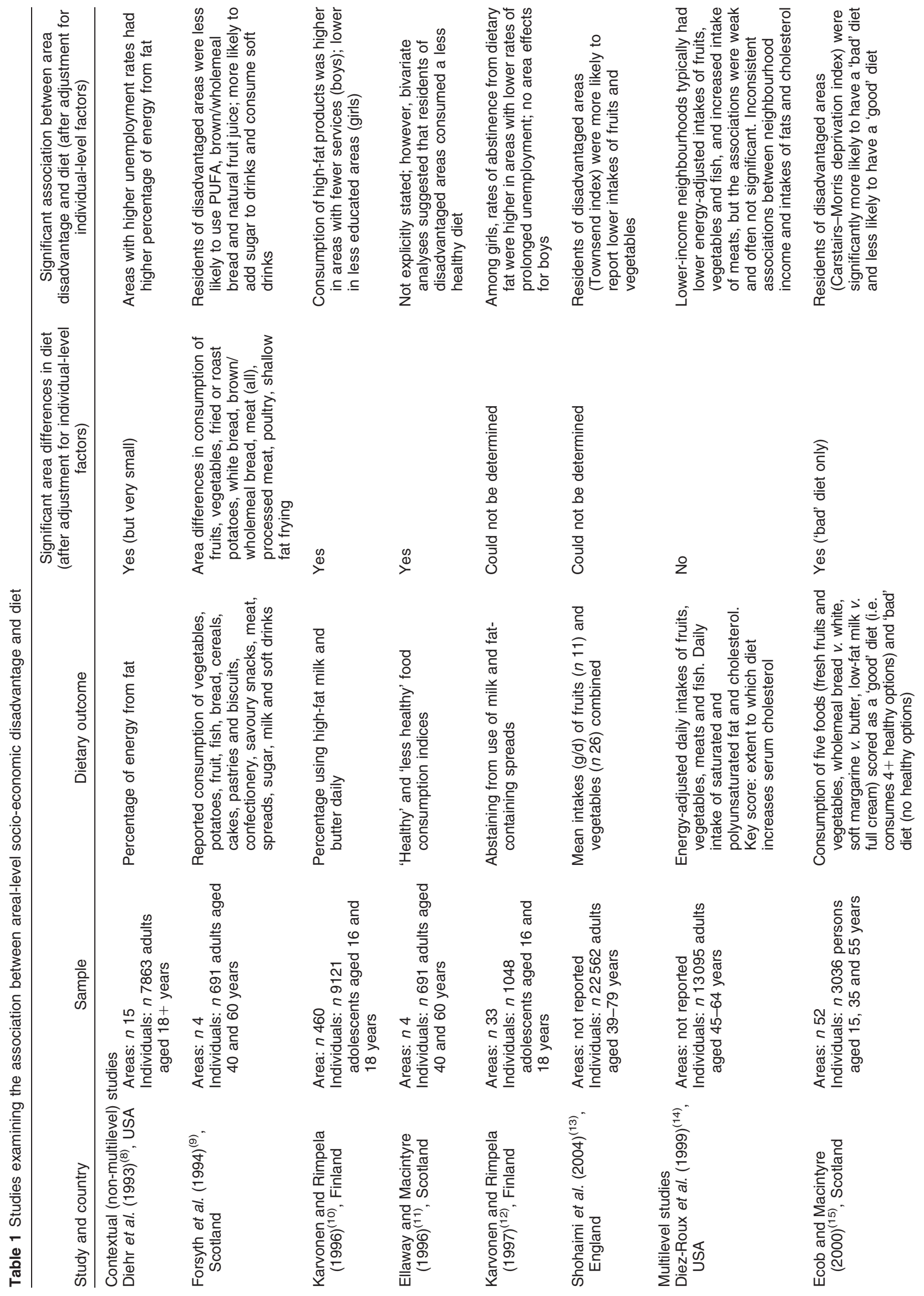


cases, the analytical approach did not allow for the partitioning of area- and individual-level sources of variation. Hence it was unclear whether differences in diet between advantaged and disadvantaged areas were due to a composition effect (i.e. the clustering of rich and poor people in rich and poor areas) or the environmental characteristics of the areas per se (i.e. a context effect, possibly reflecting area differences in physical infrastructure, services and facilities). The findings of the multilevel studies, which allow for area- and individuallevel variation to be partitioned and quantified, present a somewhat different picture. Of the five identified, only two reported a significant difference in diet between areas after adjustment for individual-level factors ${ }^{(15,17)}$. Diez-Roux et al. ${ }^{(14)}$ and Ecob and Macintyre ${ }^{(15)}$ found that residents of socio-economically disadvantaged areas had poorer diets than those in more advantaged areas, although the findings of the former were weak and often not statistically significant. Area socio-economic status (SES) was not associated with food purchasing behaviour in an Australian study ${ }^{(16)}$ or with dietary intake and food choice in a Dutch study ${ }^{(18)}$.

The present paper contributes to the literature on areas and diet by reporting on a multilevel study that examined the association between area SES and food purchasing behaviour in the Melbourne metropolitan region (Australia) in 2003. The relationship between SES and diet in Australia (and elsewhere) has to date been investigated primarily using 'quantitative' dietary indicators such as mean daily intakes of nutrients, nutrient density levels or percentage contribution of food to nutrition and energy $^{(5)}$. By contrast, few studies have examined the relationship using 'qualitative' indicators such as food purchasing behaviour. Clearly, people need to procure food (which usually means purchase it) before it can be consumed and converted into energy and nutrients, and there are a number of compelling reasons why it is important to better understand the factors that influence the food purchasing choices of different socio-economic groups. First, most people make dietary decisions in relation to food and not nutrients ${ }^{(19)}$; thus when shopping food choices are more likely to be influenced by factors such as price, availability, taste preference and convenience than by the vitamin and mineral content of the food. Second, research has shown that the type of food people buy influences dietary quality ${ }^{(20)}$. Third, food choice differences between socio-economic groups translate into concomitant differences in nutrient intake ${ }^{(21,22)}$. Fourth, knowing about the factors that influence socio-economic differences in food purchasing is important in assessing the reach and impact of health promotion messages, many of which focus on encouraging people to make healthy food choices when shopping $^{(23-25)}$.

The study investigates whether residents of socio-economically advantaged and disadvantaged areas differ in 
their purchase of grocery foods, fruits and vegetables. Specifically, three questions are examined:

1. Do areas vary in their food purchasing profiles?

2. To what extent does within-area clustering by individual-level SEP account for any observed differences between areas in their food purchasing profiles?

3. What is the relationship between area SES and food purchasing after adjustment for within-area differences in food purchasing by individual-level SEP?

\section{Methods}

\section{Geographic scope}

The present paper is based on data collected as part of the Victorian Lifestyle and Neighbourhood Environment Study (VicLANES), a cross-sectional multilevel investigation of area- and individual-level factors and health-related behaviour. The target population for VicLANES comprised people living in an area extending $20 \mathrm{~km}$ from the central business district of Melbourne city, the capital of the state of Victoria.

\section{Sample design}

The sample comprised non-institutionalised residents of private dwellings (households) and Census Collector Districts (CCD). A CCD is the smallest administrative unit used by the Australian Bureau of Statistics to collect census data. In urban areas such as Melbourne, a CCD contains an average of 200 private dwellings which are deemed to be relatively homogeneous in terms of their socio-economic characteristics. Households and CCD were selected using a stratified two-stage cluster design. At the first stage, all CCD in the Melbourne metropolitan area were ranked according to the proportion of households in each CCD with an income of less than \$AUS 400 per week. The resultant distribution was stratified into septiles, and a total of fifty CCD were randomly selected from the low- ( $n 17)$, middle( $n$ 16) and high-income ( $n$ 17) strata. At the second stage, we used names and addresses on the Australian Electoral Roll to identify all residents aged 18-74 years in each of the fifty CCD. Voting is compulsory in Australia for persons aged 18 years and over, so the electoral roll provides nearcomplete coverage of the resident adult population. A total of 3995 households were then randomly sampled, and the person within each household who was primarily responsible for most of the food shopping was targeted for data collection.

\section{Data collection}

The household-level data collection within each CCD occurred between September and December 2003, and was conducted using a mail-survey method described by Dillman $^{(26)}$. A total of 2564 usable surveys were returned to yield a final response rate of $64 \cdot 2 \%$.

\section{Measures}

Area socio-economic status

The septiles forming the sampling strata were used as the basis for measuring area SES. In each of the three strata the average proportion of households earning less than \$AUS 400 per week was $7 \cdot 0 \%$ (range 3.5-8.5\%), 15.3\% $(14 \cdot 4-16 \cdot 7 \%)$ and $31 \cdot 0 \%(24 \cdot 1-59 \cdot 6 \%)$, respectively; these strata were subsequently labelled as high, medium and low SES. The area-level socio-economic characteristics of the three strata were further examined using 2001 census data ${ }^{(27)}$, and they differed markedly in terms of their unemployment rate $(4.0 \%, 6.6 \%$ and $11.0 \%$, respectively), the percentage of employees in unskilled and semi-skilled jobs $(7 \cdot 1 \%, 13 \cdot 8 \%$ and $20 \cdot 7 \%$, respectively), the percentage of dwellings that were rented from the public housing authority $(0 \cdot 17 \%, 1 \cdot 6 \%$ and $14 \cdot 5 \%$, respectively) and the percentage of dwellings with no motor vehicle $(3 \cdot 9 \%, 9 \cdot 6 \%$ and $21 \cdot 2 \%$, respectively).

\section{Education}

Respondents were asked to provide information about whether they had attained further education since leaving school and, if so, the highest qualification completed. Respondent's education was subsequently coded as: (i) bachelor degree or higher (the latter included postgraduate diploma, master's degree or doctorate); (ii) diploma (associate or undergraduate); (iii) vocational (trade or business certificate, or apprenticeship); and (iv) no post-school qualifications.

\section{Occupation}

Respondents who were employed at the time of completing the survey were asked to indicate their job title and then to describe the main tasks or duties they performed. This information was subsequently coded to the Australian Standard Classification of Occupations (ASCO) ${ }^{(28)}$. For the purposes of the present study, the original nine-level ASCO classification was re-coded into three categories: (i) managers/professionals (managers and administrators, professionals and para-professionals); (ii) white-collar employees (clerks, salespersons and personal service workers); and (iii) blue-collar employees (trades-persons, plant and machine operators and drivers, labourers and related workers). A fourth category, 'not in the labour force', comprising the retired, unemployed, students and those engaged in home duties on a full-time basis, was also created.

\section{Income}

Respondents were asked to indicate their total annual household income (including pensions, allowances and investments) using a fourteen-category measure that was subsequently re-coded into five groups for analysis: (i) \$AUS 78000 or more; (ii) \$AUS 52000-77999; (iii) \$AUS 36 400-51 999; (iv) \$AUS 20 800-36399; and (v) less than \$AUS 20799. Households in categories (iv) and 
(v) received annual incomes at or below the Australian average in $2000^{(29)}$.

\section{Confounding}

Age in years (centred), sex and household composition were used as potential confounding variables.

\section{Food purchasing}

As part of the questionnaire, information was sought about the purchase of grocery items, fruits and vegetables.

Grocery food purchase. This was examined on the basis of fifteen questions, each of which had two or more response options. For example, respondents were asked 'When shopping for your household, what type of milk do you usually buy?' The response options included: 'I do not buy milk', 'extra creamy', 'full cream', 'low-fat/trim', 'skimmed/fat-free', plus others. Multiple responses were permitted for each question. The other fourteen questions were structured in an identical manner and pertained to bread, rice, pasta, noodles, baked beans, tinned fruit, cheese, yoghurt, beef mince, chicken, tinned fish, cooking oils, butter and solid cooking fat. In Australia, dietary authorities recommend that people purchase and consume a variety of foods that are relatively high in fibre and low in fat, salt and sugar ${ }^{(25)}$; consistent with these guidelines, we classified the foods into a recommended and regular category (Table 2). Using this classification, we developed a measure that captured the extent to which peoples' grocery purchasing patterns were consistent (or not) with dietary guideline recommendations. First, for each food type (e.g. milk), respondents were assigned the value 1 if they reported usually purchasing only the regular option exclusively (and not any recommended options); they were assigned the value 3 if they reported usually purchasing only the recommended option exclusively (and not any regular options); and they were assigned a value of 2 if they reported usually purchasing a mix of regular and recommended options (e.g. full cream and skimmed milk). There was a small number of respondents who reported that they never purchased a particular type of food and these were assigned the value 0 . In sum, for each of the fifteen food types, respondents were assigned a value of $0,1,2$ or 3 . Second, an initial food purchasing index was created that involved summing the scores for the fifteen food types, with those scoring 0 being excluded at this point. This initial index had a potential range of $15-45$, with 15 denoting people who purchased the regular option for each food type and 45 denoting those who purchased the recommended option for all foods. It is important to note that the respondents included in this initial index reported purchasing all of the fifteen food types. Those scoring 0 for one or more food type(s) were excluded because their final index score would not accurately reflect their purchasing pattern. For example, someone who purchased all fifteen food types and chose the recommended option for each item would score 45, whereas someone who purchased thirteen food types and chose the recommended option for each item would score 39. Clearly, both people have identical purchasing patterns with respect to the dietary guidelines (i.e. they are making the healthier choice for every food item) but this is not reflected in their index score. To deal with this issue, and as a way of including the full sample in the analyses, respondents who reported not buying one or more of the food items were included in the index using the following formula: Index score $=a /(15-b)$. The quantity $a$ represented each respondent's initial score which was derived by summing the values (1, 2 or 3 ) for each of the food types. The denominator comprised the constant ' 15 ', which represented the number of food types in the index, and the variable $b$, which represented the number of food

Table 2 Classification of grocery food types into 'recommended' and 'regular' categories

\begin{tabular}{|c|c|c|}
\hline Food type & Recommended & Regular \\
\hline Bread & $\begin{array}{l}\text { Wholemeal, multigrain, white high in fibre, rye, soya } \\
\text { and linseed }\end{array}$ & White \\
\hline Rice & Wholemeal or brown & Plain white and other white rice (basmati, jasmine, Arborio) \\
\hline Pasta & Wholemeal or brown & Other pasta (white, spinach, herb) \\
\hline Noodles & Wholemeal or brown & Other noodles (white, egg, spinach) \\
\hline Baked beans & Salt-reduced or unsalted & Regular salt \\
\hline Tinned fruit & In natural juice & In syrup \\
\hline Cheese & Reduced fat (25\% less fat), low-fat (<10\% fat) & Full-fat \\
\hline Milk & $\begin{array}{l}\text { Reduced fat, low-fat, high-Ca, high-Ca skimmed, } \\
\text { high-Fe, high-protein, reduced lactose, no } \\
\text { cholesterol, soya or soya \& linseed (skimmed) }\end{array}$ & Extra creamy, full cream, soya or soya \& linseed (full cream) \\
\hline Yoghurt & Low-fat (plain and fruit) & Full-fat (plain and fruit) \\
\hline Beef mince & Lean (trim/premium) & Regular (choice/fine grade) \\
\hline Chicken (uncooked) & Without skin, with skin (and remove before eating) & With skin (and eat skin) \\
\hline Tinned fish & In water/spring water & In oil or brine \\
\hline Vegetable oil & $\begin{array}{l}\text { Canola, sunflower, safflower, olive, corn, soyabean, } \\
\text { peanut or sesame, grape seed or macadamia }\end{array}$ & Blended oils, coconut oil, palm oil \\
\hline Butter & Salt-reduced, unsalted & Regular salt \\
\hline Solid cooking fat & Cooking margarine, solidified oil & $\begin{array}{l}\text { Solid animal fat (lard, beef dripping), vegetable shortening, } \\
\text { ghee or butter (and use for cooking) }\end{array}$ \\
\hline
\end{tabular}


types not purchased by the respondent. In effect, the formula calculated a mean food purchasing score for each respondent. Finally, the index was re-scored to range from 0 to 100, with higher scores indicating a purchasing pattern that was more consistent with dietary guideline recommendations (sample mean $47 \cdot 6$, sD $13 \cdot 4$ ).

Fruit purchasing. This was examined using a question that asked 'When shopping for fresh fruit, how often do you buy these types?' The respondent was instructed to include seasonal fruits, but exclude fruit juice, canned and dried fruit. The question item-set consisted of twentytwo fresh fruits selected (mostly) from the FFQ used in the 1995 Australian National Nutrition Survey ${ }^{(30)}$. For each fruit, respondents were asked to indicate their usual purchasing pattern on the basis of five-point scales: $1=$ 'never buy', $2=$ 'rarely buy', $3=$ 'sometimes buy', $4=$ 'nearly always buy' and $5=$ 'always buy'.

Using these items we created an index that measured variety of fruit purchased. For each fruit item, respondents reporting 'never' or 'rarely' buy were scored 0 , and those reporting any of the other three options were scored 1 . The items were then summed, with the resultant index score for each respondent indicating the variety of fruits purchased (sample mean 14.2, SD 4·1). Importantly, the variety score does not reflect the range of fruits purchased on any particular shopping trip, but rather the types that are purchased at least sometimes over the course of many shopping episodes depending on factors such as seasonality, price and quality. As the variety index was essentially a count-measure and nonnormally distributed it was categorised into quartiles, with Q1 denoting high variety and Q4 low variety.

Vegetable purchasing. Respondents were asked to indicate how often they purchased twenty-five vegetables, including fresh and frozen, but excluding canned or dried vegetables. A purchasing index measuring vegetable variety was constructed using an identical format and method to that used for fresh fruit. The mean variety score for vegetables for the sample was $18 \cdot 5$ (SD $4 \cdot 1$ ).

\section{Analysis}

Table 3 presents descriptive statistics for each of the measures used in this analysis.

From the 2564 questionnaires that were returned, missing data were identified for education ( $n 106,4 \cdot 1 \%$ ), occupation ( $n 83,3 \cdot 2 \%)$, income ( $n 903,35 \cdot 2 \%)$, sex ( $n 4$, $0 \cdot 16 \%)$, age $(n 5,0 \cdot 20 \%)$ and household composition ( $n 55,2 \cdot 1 \%$ ). In total, the proportion of the sample with completely observed data for all the variables examined (complete cases) was $57 \%$. We have not reported results obtained by analysing only the complete cases because of the potential bias and loss of precision associated with the large proportion of missing income data; instead, we used multiple imputation. We imputed all missing data under a missing at random (MAR) assumption and adopted an inclusive strategy for the imputation model ${ }^{(31-33)}$.
Table 3 Descriptive statistics for the socio-economic and demographic variables and the measures of food purchasing behaviour (Melbourne city, Australia, 2003; $n$ 2564)

\begin{tabular}{|c|c|c|}
\hline & $n$ & $\%$ \\
\hline \multicolumn{3}{|l|}{ Area disadvantage } \\
\hline Low & 914 & $35 \cdot 7$ \\
\hline Medium & 895 & 34.9 \\
\hline High & 755 & $29 \cdot 5$ \\
\hline \multicolumn{3}{|l|}{ Education } \\
\hline Bachelor degree or higher & 815 & $31 \cdot 8$ \\
\hline Diploma & 290 & $11 \cdot 3$ \\
\hline Vocational & 393 & $15 \cdot 3$ \\
\hline No post-school qualifications & 1006 & $41 \cdot 6$ \\
\hline \multicolumn{3}{|l|}{ Occupation } \\
\hline Professionals & 861 & $33 \cdot 6$ \\
\hline White collar & 485 & $18 \cdot 9$ \\
\hline Blue collar & 140 & $5 \cdot 5$ \\
\hline Not in the labour force & 1078 & $42 \cdot 0$ \\
\hline \multicolumn{3}{|l|}{ Annual income } \\
\hline \$AUS 78000 or more & 702 & $27 \cdot 4$ \\
\hline \$AUS $52000-77999$ & 605 & $23 \cdot 6$ \\
\hline \$AUS $36400-51999$ & 398 & $15 \cdot 5$ \\
\hline \$AUS 20 800-36 399 & 391 & $15 \cdot 3$ \\
\hline \$AUS 20799 or less & 468 & $18 \cdot 3$ \\
\hline \multicolumn{3}{|l|}{ Sex } \\
\hline Female & 2181 & $85 \cdot 1$ \\
\hline Male & 383 & $14 \cdot 9$ \\
\hline \multicolumn{3}{|l|}{ Household composition } \\
\hline 1 adult, no children & 427 & $16 \cdot 7$ \\
\hline 1 adult, 1 or more children & 207 & $8 \cdot 1$ \\
\hline 2 or more adults, no children & 911 & $35 \cdot 5$ \\
\hline \multirow[t]{2}{*}{2 or more adults, 1 or more children } & 1019 & $39 \cdot 7$ \\
\hline & Mean & SD \\
\hline Age (years) & $49 \cdot 0$ & $13 \cdot 5$ \\
\hline
\end{tabular}

Five data sets with imputed values for missing items on each variable were estimated using the command 'Imputation by Chained Equations (ICE)' in the STATA statistical software package version 9·2 (Stata Corporation, College Station, TX, USA).

The grocery data were analysed as a two-level random intercept model in STATA. We specified three models that directly addressed the three research questions identified earlier. Model 1 (baseline) quantified the extent of arealevel variation in food purchasing behaviour conditional on the confounders. Here, the substantive interest was on the random term which, if significant, indicated that food purchasing patterns differed between the fifty CCD. For this and subsequent models we also calculated an intraclass correlation (ICC) by dividing the between-CCD variance by the total variance, and this is interpreted as the proportion of the total variation in food purchasing behaviour that is between the CCD. Model 2 extends Model 1 by adding education, occupation and income as fixed effects, and examined the extent to which they account for variation in food purchasing between the CCD. Model 3 then extended Model 2 by including the measure of area SES as a fixed effect; here the focus is on whether area SES is associated with food purchasing independently of within-area variation in age, sex, household composition and individual-level SEP. 
Variety of fruit and vegetable purchase was examined using a two-level ordered multinomial logit-link model. 'High' variety (Q1) was denoted the reference category; hence positive regression coefficients for any of the predictor variables indicate a greater odds of purchasing a lower variety of fruits and vegetables. Three models were specified. Model 1 (baseline) quantified the extent of area-level variation in fruit and vegetable variety conditional on the confounders. Model 2 added education, occupation and income, and Model 3 included area SES. The results are presented as odds ratios and their 95\% confidence intervals.

\section{Results}

Table 4 presents the findings of the multilevel analyses which examined the independent contribution of area- and individual-level socio-economic factors to grocery food purchase. In Model 1, the area-level random term was statistically significant $(P=0 \cdot 033)$, indicating that the average grocery purchasing score was different (beyond chance) across the fifty CCD. Of the total variability in grocery purchase, $1.5 \%$ occurred between CCD and $98.5 \%$ between individuals. Model 2 adds the fixed (average) effects for education, occupation and income; this attenuated the between-area variation by $59 \cdot 8 \%$, and the random term was no longer significant $(P=0 \cdot 241)$. Education and income were associated with grocery purchase: respondents with no post-school qualifications and those living in low-income households scored significantly lower on the index. No significant occupational effects were observed. Model 3 adds the fixed effect for area SES and the coefficients indicate that residents of medium- and low-SES areas scored significantly lower on the grocery purchasing index than their counterparts from high-SES areas.

Table 5 presents the findings of the ordered multilevel logistic regression analysis which examined the contribution of area- and individual-level socio-economic factors to variety of fruit and vegetable purchasing. Fruit variety scores were significantly different $(P=0 \cdot 01)$ across the fifty CCD (Model 1 ). After adjustment for education, occupation and income (Model 2), the between-area variation in fruit variety was attenuated by $50.0 \%$ and remained marginally statistically significant $(P=0 \cdot 06)$. Respondents with no post-school qualifications had 1.72 (95\% CI $1 \cdot 25,2 \cdot 38)$ times higher odds of purchasing a lower variety of fruits. The corresponding odds for respondents

Table 4 Area- and individual-level socio-economic effects on the purchase of grocery foods consistent with dietary guideline recommendations (Melbourne city, Australia, 2003)

\begin{tabular}{|c|c|c|c|c|c|c|}
\hline & \multicolumn{6}{|c|}{ Groceriest } \\
\hline & \multicolumn{2}{|c|}{ Model 1} & \multicolumn{2}{|c|}{ Model 2} & \multicolumn{2}{|c|}{ Model 3} \\
\hline & $\beta$ & SE & $\beta$ & SE & $\beta$ & SE \\
\hline Intercept & $43 \cdot 0$ & $1 \cdot 5$ & $41 \cdot 5$ & $1 \cdot 1$ & $42 \cdot 8$ & $1 \cdot 1$ \\
\hline \multicolumn{7}{|l|}{ Area SES } \\
\hline High & & & & & - & \\
\hline Medium & & & & & $-2 \cdot 09$ & $0 \cdot 70^{\star *}$ \\
\hline Low & & & & & $-2 \cdot 43$ & $0 \cdot 76^{\star \star \star}$ \\
\hline \multicolumn{7}{|l|}{ Education } \\
\hline Bachelor degree or higher & & & - & & - & \\
\hline Diploma & & & $0 \cdot 35$ & 0.99 & $0 \cdot 45$ & 0.98 \\
\hline Vocational & & & $0 \cdot 22$ & 0.93 & $0 \cdot 20$ & 0.93 \\
\hline No post-school qualifications & & & $-1 \cdot 73$ & $0 \cdot 79^{\star *}$ & $-1 \cdot 54$ & $0 \cdot 78^{*}$ \\
\hline \multicolumn{7}{|l|}{ Occupation } \\
\hline Professionals & & & - & & - & \\
\hline White collar & & & -0.09 & $0 \cdot 89$ & -0.07 & $0 \cdot 88$ \\
\hline Blue collar & & & 0.41 & $1 \cdot 38$ & 0.64 & $1 \cdot 37$ \\
\hline Not in the labour force & & & $-0 \cdot 85$ & $0 \cdot 80$ & -0.83 & $0 \cdot 80$ \\
\hline \multicolumn{7}{|l|}{ Annual income } \\
\hline \$AUS 78000 or more & & & - & & - & \\
\hline \$AUS $52000-77999$ & & & $-0 \cdot 86$ & $0 \cdot 81$ & $-0 \cdot 70$ & $0 \cdot 80$ \\
\hline \$AUS $36400-51999$ & & & $-1 \cdot 08$ & 0.94 & $-0 \cdot 78$ & 0.93 \\
\hline \$AUS 20800-36399 & & & $-2 \cdot 47$ & $0 \cdot 99^{\star}$ & $-2 \cdot 06$ & $0.99^{*}$ \\
\hline \$AUS 20799 or less & & & $-2 \cdot 98$ & $1 \cdot 05^{\star *}$ & $-2 \cdot 31$ & $1 \cdot 06^{\star}$ \\
\hline \multicolumn{7}{|l|}{ Random effects } \\
\hline Area variance & $2 \cdot 54$ & $1 \cdot 2$ & 1.02 & 0.9 & $0 \cdot 182$ & $0 \cdot 7$ \\
\hline$P$ value for area variance & \multicolumn{2}{|c|}{$\begin{array}{l}0.033 \\
1.5\end{array}$} & \multicolumn{2}{|c|}{$0 \cdot 241$} & \multicolumn{2}{|c|}{$0 \cdot 784$} \\
\hline Intra-class correlation (\%) & & & & & & \\
\hline
\end{tabular}

SES, socio-economic status.

Effect was significant: ${ }^{\star} P \leq 0 \cdot 05,{ }^{* \star} P \leq 0 \cdot 01,{ }^{* \star *} P \leq 0 \cdot 001$.

tModel 1, baseline model adjusted for age, sex and household composition; Model 2, Model 1 plus education, occupation and income; Model 3 , Model 2 plus area SES. 
Table 5 Area- and individual-level socio-economic effects on variety of fruit and vegetable purchasing (Melbourne city, Australia, 2003)†

\begin{tabular}{|c|c|c|c|c|c|c|c|c|c|c|}
\hline & \multicolumn{5}{|c|}{ Fruit variety $\ddagger$} & \multicolumn{5}{|c|}{ Vegetable variety } \\
\hline & \multirow[t]{2}{*}{ Model 1} & \multicolumn{2}{|c|}{ Model 2} & \multicolumn{2}{|c|}{ Model 3} & \multirow[t]{2}{*}{ Model 1} & \multicolumn{2}{|c|}{ Model 2} & \multicolumn{2}{|c|}{ Model 3} \\
\hline & & OR & $95 \% \mathrm{Cl}$ & OR & $95 \% \mathrm{Cl}$ & & OR & $95 \% \mathrm{Cl}$ & OR & $95 \% \mathrm{Cl}$ \\
\hline \multicolumn{11}{|l|}{ Area SES } \\
\hline High & & & & $1 \cdot 00$ & & & & & $1 \cdot 00$ & \\
\hline Medium & & & & 1.07 & $0 \cdot 84,1 \cdot 37$ & & & & $0 \cdot 88$ & $0 \cdot 70,1 \cdot 11$ \\
\hline Low & & & & $1 \cdot 30$ & $1 \cdot 00,1 \cdot 67$ & & & & $1 \cdot 06$ & $0.83,1 \cdot 35$ \\
\hline \multicolumn{11}{|l|}{ Education } \\
\hline Bachelor degree or higher & & $1 \cdot 00$ & & $1 \cdot 00$ & & & $1 \cdot 00$ & & $1 \cdot 00$ & \\
\hline Diploma & & $1 \cdot 27$ & $0 \cdot 84,1 \cdot 91$ & $1 \cdot 26$ & $0.94,1.68$ & & $1 \cdot 08$ & $0 \cdot 78,1 \cdot 48$ & 1.07 & $0 \cdot 79,1 \cdot 45$ \\
\hline Vocational & & $1 \cdot 44$ & $0 \cdot 99,2 \cdot 11$ & $1 \cdot 45$ & $1 \cdot 11,1 \cdot 89$ & & $1 \cdot 01$ & $0 \cdot 75,1 \cdot 36$ & $1 \cdot 01$ & $0 \cdot 76,1 \cdot 34$ \\
\hline No post-school qualifications & & $1 \cdot 72$ & $1 \cdot 25,2 \cdot 38$ & $1 \cdot 70$ & $1 \cdot 35,2 \cdot 14$ & & $1 \cdot 36$ & $1 \cdot 06,1 \cdot 75$ & $1 \cdot 36$ & $1 \cdot 08,1.72$ \\
\hline \multicolumn{11}{|l|}{ Occupation } \\
\hline Professionals & & $1 \cdot 00$ & & $1 \cdot 00$ & & & $1 \cdot 00$ & & $1 \cdot 00$ & \\
\hline White collar & & 0.85 & $0.59,1 \cdot 22$ & 0.85 & $0 \cdot 66,1 \cdot 10$ & & $1 \cdot 02$ & $0 \cdot 77,1 \cdot 35$ & $1 \cdot 02$ & $0.78,1 \cdot 33$ \\
\hline Blue collar & & 0.94 & $0.54,1 \cdot 64$ & 0.93 & $0 \cdot 62,1 \cdot 37$ & & $1 \cdot 14$ & $0 \cdot 74,1 \cdot 76$ & $1 \cdot 15$ & $0 \cdot 76,1 \cdot 73$ \\
\hline Not in the labour force & & 0.91 & $0 \cdot 66,1 \cdot 27$ & 0.91 & $0 \cdot 72,1 \cdot 15$ & & $1 \cdot 13$ & $0 \cdot 88,1 \cdot 46$ & $1 \cdot 12$ & $0 \cdot 88,1 \cdot 43$ \\
\hline \multicolumn{11}{|l|}{ Annual income } \\
\hline \$AUS 78000 or more & & $1 \cdot 00$ & & $1 \cdot 00$ & & & $1 \cdot 00$ & & $1 \cdot 00$ & \\
\hline \$AUS 52 000-77999 & & $1 \cdot 26$ & $0 \cdot 90,1 \cdot 75$ & $1 \cdot 25$ & $0.99,1.58$ & & $0 \cdot 19$ & $0.93,1.56$ & $1 \cdot 21$ & $0.95,1.55$ \\
\hline \$AUS $36400-51999$ & & 1.09 & $0 \cdot 74,1 \cdot 60$ & 1.07 & $0 \cdot 81,1 \cdot 40$ & & $0 \cdot 88$ & $0 \cdot 65,1 \cdot 18$ & $0 \cdot 88$ & $0 \cdot 66,1 \cdot 17$ \\
\hline \$AUS 20800-36 399 & & $1 \cdot 43$ & $0 \cdot 95,2 \cdot 13$ & $1 \cdot 39$ & $1 \cdot 04,1 \cdot 85$ & & $1 \cdot 12$ & $0 \cdot 81,1 \cdot 53$ & $1 \cdot 12$ & $0.83,1.52$ \\
\hline \$AUS 20799 or less & & $1 \cdot 69$ & $1 \cdot 11,2 \cdot 57$ & $1 \cdot 59$ & $1 \cdot 18,2 \cdot 16$ & & $1 \cdot 17$ & $0 \cdot 84,1 \cdot 63$ & $1 \cdot 16$ & $0 \cdot 84,1 \cdot 60$ \\
\hline \multicolumn{11}{|l|}{ Random effects } \\
\hline Area variance and SE & $0.08 \quad 0.03$ & 0.04 & 0.02 & 0.04 & $0 \cdot 02$ & $0.03 \quad 0.02$ & 0.02 & 0.02 & 0.01 & 0.02 \\
\hline$P$ value for area variance & 0.01 & \multicolumn{2}{|c|}{0.06} & \multicolumn{2}{|c|}{$0 \cdot 11$} & $0 \cdot 11$ & \multicolumn{2}{|c|}{0.30} & \multicolumn{2}{|c|}{0.41} \\
\hline
\end{tabular}

SES, socio-economic status.

tHigh variety (quartile 1) was denoted the reference category; hence odds ratios greater than 1 indicate an increased likelihood of purchasing a lower variety of fruits and vegetables.

¥Model 1, baseline model adjusted for age, sex and household composition; Model 2, Model 1 plus education, occupation and income; Model 3 , Model 2 plus area SES.

from low-income families were $1 \cdot 69(95 \%$ CI $1 \cdot 11,2 \cdot 57)$. Model 3 adds the measure of area SES which made no appreciable difference to the between-CCD variation (relative to Model 2) although the random term was no longer statistically significant $(P=0 \cdot 11)$. The coefficients for area SES show that residents of low-SES areas had significantly higher odds of purchasing a lower variety of fruits than residents in the high-SES areas $(\mathrm{OR}=1 \cdot 30,95 \% \mathrm{CI} 1 \cdot 00$, 1.67). Independent of area SES, respondents with lower levels of education, and residents of lower-income households, had significantly higher odds of purchasing a more limited variety of fruits than their higher status counterparts.

Vegetable variety scores did not differ significantly across the fifty CCD (Model 1) and the inclusion of education, occupation and income further attenuated the CCD variation (Model 2). Respondents with no postschool qualifications had a significantly higher odds of purchasing a lower variety of vegetables relative to those with a bachelor degree $(\mathrm{OR}=1 \cdot 36,95 \% \mathrm{CI} 1 \cdot 08,1 \cdot 72)$. There was no association between vegetable variety and occupation, income or area SES (Model 3).

\section{Discussion}

In metropolitan Melbourne in 2003, area SES was associated with the purchase of grocery foods and fruit variety.
Compared with their counterparts in high-SES areas, residents of low-SES areas were less likely to buy groceries that were high in fibre and low in fat, salt and sugar; and they purchased a smaller variety of fruits. These findings are broadly consistent with the results of multilevel studies conducted in the USA ${ }^{(14)}$ and Scotland ${ }^{(15)}$; however, they are at odds with multilevel research conducted in The Netherlands ${ }^{(18)}$ and Brisbane, Australia ${ }^{(16)}$. Reconciling these differences, and hence being able to generalise about the relationship between area SES and diet, is difficult. In part, these difficulties stem from the limited evidence base (i.e. the small number of multilevel studies) and methodological issues such as differences in the conceptualisation and measurement of diet, the individual-level variables used as confounders, and the number and size of the area units used ${ }^{(15)}$. The inconsistencies between study findings, however, are likely to be more than a methodological artefact, and may reflect 'real' historical, cultural, political, socio-economic and geospatial differences between countries (e.g. USA and Australia) and between regions within the same country (e.g. Brisbane and Melbourne). At present, the mixed findings of the small number of multilevel studies do not provide a sufficiently reliable basis on which to make a general call for area-level public health interventions to improve conditions in deprived areas to facilitate the procurement of foods that are conducive to a healthy diet; rather, any 'call' 
may have to be specific and tailored to each particular geographic and spatial context.

A large literature documents an association between individual-level SEP and diet, and most of this work has focused on socio-economic differences in food and nutrient intakes ${ }^{(5)}$. These studies usually find that socioeconomically disadvantaged groups have intakes that are consistent with their higher rates of diet-related chronic disease $^{(1-3)}$. To some extent at least, the results of the present food purchasing study extend and complement the findings of the intake studies by showing that those of low SEP are less likely to buy grocery foods that accord with diet-related health promotion messages and dietary guidelines. In addition, low socio-economic groups had significantly higher odds of purchasing a lower variety of fruits and vegetables.

\section{Study limitations}

First, survey non-response tends to be higher in disadvantaged areas $^{(34)}$ and among individuals of low SEP ${ }^{(35)}$. Non-response in the VicLANES study was 35.8\%; hence the sample probably under-represents the disadvantaged areas and individuals and over-represents the advantaged, and the observed socio-economic differences in food purchasing are likely to be an underestimate of the actual differences in the Melbourne population.

Second, as with most multilevel studies ${ }^{(36)}$, our use of a CCD to represent a neighbourhood was made for reasons of sampling and analytic convenience rather than being underpinned by an explicit theory linking area SES and food purchasing; hence associations among these variables are likely to be underestimated.

Third, our finding of an association between area SES and food purchase might be confounded by individuallevel socio-economic factors not included in the models. This said however, we included the three most widely used indicators of a person's socio-economic characteristics ${ }^{(37)}$ and, given the correlation among these indicators $^{(38)}$, it is likely that education, occupation and income were capturing most of the unmeasured influences of other socio-economic factors excluded from the models. Alternatively, it may be that the inclusion of these individual-level measures resulted in 'over-adjustment', which argues for the possibility of an even stronger contextual effect on food purchase than was observed here. If education, occupation and household income represent part of the pathway via which area SES influences food procurement, then modelling individual-level socio-economic variables may inappropriately attenuate the variation that is more correctly attributable to area disadvantage ${ }^{(39)}$.

\section{Conclusion}

In the Melbourne metropolitan region in 2003, differences between advantaged and disadvantaged areas in their purchasing profiles for grocery foods and fruits, and the 'healthier' purchasing in higher-SES areas, suggest that the areas may be differentiated on the basis of food availability, accessibility and affordability, making the purchase of some types of foods more difficult for people living in disadvantaged areas. To date, the between- and withincountry (multilevel) evidence linking area disadvantage and diet is both sparse and inconsistent. Methodological issues notwithstanding, this might suggest that area deprivation is not universally associated with poorer access to healthy food. Cummins and Macintyre ${ }^{(40)}$ reached a somewhat similar conclusion based on their review of the literature on food environments and obesity. A challenge for future area-based dietary research is to identify those ecological characteristics (e.g. urban design, shopping infrastructure, transport services) that promote equality of access to healthy food, and those characteristics that make its attainment difficult.

\section{Acknowledgements}

The study was supported by a grant from the Victorian Health Promotion Foundation (VicHealth). G.T. is supported by a National Health and Medical Research Council (NHMRC) Senior Research Fellowship (No. 390109); R.B. and L.R.T. by an NHMRC Capacity Building Grant; and S.V.S. is supported by a National Institutes of Health Career Development Award (NHLBI K25 HL081275). There are no conflicts of interest. G.T. conceptualised the paper and played the lead role in writing the manuscript and reviewing the literature. R.B. contributed to the data analysis and imputation (revised submission) and to writing the Methods section. L.R.T. contributed to the data analysis and imputation (initial submission) and editing the manuscript. D.J. provided statistical advice, undertook preliminary analysis and contributed to writing and checking the manuscript. S.V.S. contributed to the conceptualisation of the analysis plan, provided statistical advice in relation to the multilevel modelling and edited the manuscript. A.M.K. contributed to the conceptualisation, analysis plan and writing. We gratefully acknowledge Mr Lukar Thornton for his assistance with the missing data imputation and Ms Tania King for her work as Project Manager on VicLANES.

\section{References}

1. Metcalf P, Scragg R \& Davis P (2006) Dietary intakes by different markers of socioeconomic status: results of a New Zealand workforce survey. $N Z$ Med J 26, U2127.

2. Shahar D, Shai L, Vardi H, Shahar A \& Fraser D (2005) Diet and eating habits in high and low socioeconomic groups. Nutrition 21, 559-566.

3. De Irala-Estevez J, Groth M, Johansson L, Oltersdorf U, Prattala R \& Martinez-Gonzalez MA (2000) A systematic review of socioeconomic differences in food habits in Europe: consumption of fruit and vegetables. Eur J Clin Nutr 54, 706-714. 
4. Turrell G (1997) Compliance with the Australian dietary guidelines in the early 1990's: have population-based health promotion programs been effective? Nutr Health 11, 271-288.

5. Turrell G, Hewitt B, Patterson C, Oldenburg B \& Gould T (2002) Socioeconomic differences in food purchasing behaviour and suggested implications for diet-related health promotion. J Hum Nutr Diet 15, 355-364.

6. James WPT, Nelson M \& Leather S (1997) The contribution of nutrition to inequalities in health. BMJ 314, 1545-1549.

7. Davey Smith G \& Brunner E (1997) Socioeconomic differentials in health: the role of nutrition. Proc Nutr Soc 56, 75-90.

8. Diehr P, Koepsell T, Cheadle A, Psaty BM, Wagner E \& Curry S (1993) Do communities differ in health behaviors? J Clin Epidemiol 46, 1141-1149.

9. Forsyth A, Macintyre S \& Anderson A (1994) Diets for disease? Intraurban variation in reported food consumption in Glasgow. Appetite 22, 259-274.

10. Karvonen S \& Rimpela A (1996) Socio-regional context as a determinant of adolescents' health behaviour in Finland. Soc Sci Med 43, 1467-1474.

11. Ellaway A \& Macintyre S (1996) Does where you live predict health related behaviours? A case study in Glasgow. Health Bull 54, 443-446.

12. Karvonen S \& Rimpela AH (1997) Urban small area variation in adolescents' health behaviour. Soc Sci Med 45, 1089-1098.

13. Shohaimi S, Welch A, Bingham S, Luben R, Day N, Wareham N \& Khaw K-T (2004) Residential area deprivation predicts fruit and vegetable consumption independently of individual educational level and occupational social class: a cross-sectional population study in the Norfolk cohort of the European Prospective Investigation into Cancer (EPIC-Norfolk). J Epidemiol Community Health 58, 686-691.

14. Diez-Roux AV, Nieto FJ, Caulfield L, Tyroler HA, Watson RL \& Szklo M (1999) Neighbourhood differences in diet: the Atherosclerosis Risk in Communities (ARIC) study. J Epidemiol Community Health 53, 55-63.

15. Ecob R \& Macintyre S (2000) Small area variations in health related behaviours; do these depend on the behaviour itself, its measurement, or on personal characteristics? Health Place 6, 261-274.

16. Turrell G, Blakely T, Patterson C \& Oldenburg B (2004) A multilevel analysis of socioeconomic (small area) differences in household food purchasing behaviour. $J$ Epidemiol Community Health 58, 208-215.

17. Ball K, Crawford D \& Mishra G (2006) Socio-economic inequalities in women's fruit and vegetable intakes: a multilevel study of individual, social and environmental mediators. Public Health Nutr 9, 623-630.

18. Giskes K, Turrell G, van Lenthe FJ, Brug J \& Mackenbach JP (2006) A multilevel study of socio-economic inequalities in food choice behaviour and dietary intake among the Dutch population: the GLOBE study. Public Health Nutr 9, 75-83.

19. Crotty P, Rutishauser I \& Cahill M (1991) From food to nutrients and back. Proc Nutr Soc Aust 16, 38.

20. Shimakawa T, Sorlie P, Carpenter MA, Dennis B, Tell GS, Watson R \& Williams D (1994) Dietary intake patterns and sociodemographic factors in the Atherosclerosis Risk in Communities Study. Prev Med 23, 769-780.

21. Smith A \& Baghurst K (1992) Public health implications of dietary differences between social status and occupational category groups. J Epidemiol Community Health $\mathbf{4 6}$, 409-416

22. Syrette JA, Baghurst KI \& Record SJ (1990) Sociodemographic determinants of the contribution of cereal foods to nutrient intake in the Australian population. Food Australia 42, 330-337.

23. Dixon H, Borland R \& Segan C (1998) Public reaction to Victoria's ' 2 Fruit 'n' 5 Veg every day' campaign and reported consumption of fruit and vegetables. Prev Med 27, 572-582.

24. Krebs-Smith SM \& Scott Kantor L (2001) Choose a variety of fruit and vegetables daily: understanding the complexities. J Nutr 131, Suppl., 487S-501S

25. Strategic Inter-Governmental Nutrition Alliance (2001) Eat Well Australia: An Agenda for Action for Public Health Nutrition 2001-2010. Canberra: National Public Health Partnership; available at http://www.health.vic.gov.au/ nhpa/resources/nu_eatwell.htm

26. Dillman DA (2000) Mail and Internet Surveys: The Tailored Design Method. Chichester: Wiley.

27. Australian Bureau of Statistics (2001) CDATA 2001. Your Census at Work (CD-ROM), 2nd release. Catalogue no. 2019.0.30.001. Canberra: Australian Bureau of Statistics.

28. Australian Bureau of Statistics (1997) Australian Standard Classification of Occupations, 2nd ed. Catalogue no. 1220.0. Canberra: Australian Government Publishing Service.

29. Australian Bureau of Statistics (2001) Income Distribution 1999-2000. Catalogue no. 6523.0. Canberra: Australian Government Publishing Service.

30. McLennan W (1998) National Nutrition Survey User's Guide. Canberra: Australian Government Publishing Service.

31. Collins LM, Schafer JS \& Kam C (2001) A comparison of inclusive and restrictive strategies in modern missing data procedures. Psychol Methods 6, 330-351.

32. Rubin DB (1996) Multiple imputation after 18+ years. J Am Stat Assoc 91, 473-489.

33. Meng XL (1994) Multiple-imputation inferences with uncongenial sources of input. Stat Sci 9, 538-573.

34. Turrell G, Sanders AE, Slade GD, Marcenes W \& Spencer AJ (2007) The contribution of area-level disadvantage and individual-level socioeconomic position to self-reported oral health. Community Dent Oral Epidemiol 35, 195-206.

35. Turrell G \& Najman JM (1995) Collecting food-related data from low socioeconomic groups: how adequate are our current research designs? Aust J Public Health 19, 410-416.

36. Boyle MH \& Wilms JD (1999) Place effects for areas defined by administrative boundaries. Am J Epidemiol 149, 577-585.

37. Dutton T, Turrell G \& Oldenburg B (2005) Measuring Socioeconomic Position in Population Health Monitoring and Health Research. Health Inequalities Monitoring Series no. 3. Brisbane: Queensland University of Technology.

38. Turrell G, Hewitt B, Patterson C \& Oldenburg B (2003) Measuring socio-economic position in dietary research: is choice of socio-economic indicator important? Public Health Nutr 6, 191-200.

39. Diez Roux AV (2004) Estimating neighbourhood health effects: the challenges of causal inference in a complex world. Soc Sci Med 58, 1953-1960.

40. Cummins S \& Macintyre S (2006) Food environments and obesity - neighbourhood or nation? Int J Epidemiol 35, 100-104. 